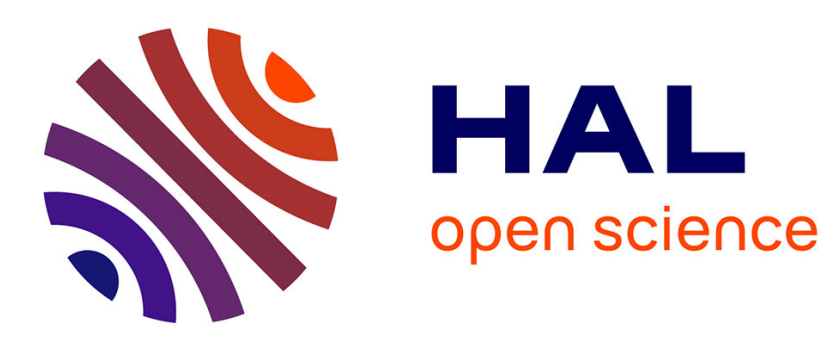

\title{
Swollen lamellar phases between two solid walls : undulation forces and generation of defects
}

\author{
O. Abillon, Emile Perez
}

\section{To cite this version:}

O. Abillon, Emile Perez. Swollen lamellar phases between two solid walls: undulation forces and generation of defects. Journal de Physique, 1990, 51 (22), pp.2543-2556. 10.1051/jphys:0199000510220254300 . jpa-00212552

HAL Id: jpa-00212552

https://hal.science/jpa-00212552

Submitted on 1 Jan 1990

HAL is a multi-disciplinary open access archive for the deposit and dissemination of scientific research documents, whether they are published or not. The documents may come from teaching and research institutions in France or abroad, or from public or private research centers.
L'archive ouverte pluridisciplinaire HAL, est destinée au dépôt et à la diffusion de documents scientifiques de niveau recherche, publiés ou non, émanant des établissements d'enseignement et de recherche français ou étrangers, des laboratoires publics ou privés. 
Classification

Physics Abstracts

$61.30-64.70-82.70$

\title{
Swollen lamellar phases between two solid walls : undulation forces and generation of defects
}

\author{
O. Abillon and E. Perez \\ Laboratoire de Physique Statistique de l'Ecole Normale Supérieure (*), 24 rue Lhomond, 75231 \\ Paris Cedex 05, France
}

(Received 31 January 1990, revised 20 June 1990, accepted 6 August 1990)

\begin{abstract}
Résumé. - Nous avons mesuré les forces entre deux surfaces de mica immergées dans une phase lamellaire gonflée à l'eau (CTAB/hexanol/eau salée). Les bicouches s'orientent parallèlement aux surfaces de mica. Les courbes force-distance présentent des domaines où l'eau est continûment éjectée d'entre les lamelles, et des domaines où le système saute rapidement à une autre distance en éjectant des lamelles. Nous avons étudié l'effet sur les forces d'un déplacement dans le diagramme de phases à période spatiale constante et également le long de la ligne de dilution. Plus on se rapproche de la transition vers la phase bicontinue $\left(\mathrm{L}_{\alpha} / \mathrm{L}_{3}\right)$, c'est-à-dire plus la concentration en hexanol est grande, plus la force nécessaire à l'éjection des lamelles devient faible. Certains effets dynamiques sont observés et peuvent s'expliquer par un couplage hydrodynamique entre les bicouches et le solvant. Ils peuvent produire des comportements surprenants: en décomprimant la phase lamellaire, des bicouches sont systématiquement éjectées. L'état d'équilibre du système est discuté. Les résultats sont en accord avec le profil en $1 / d^{2}$ calculé par Helfrich pour les forces d'ondulation. L'ajustement des courbes force-distance à une loi en $1 / d^{2}$ nous donne une valeur de la rigidité de courbure des lamelles : $k_{\mathrm{c}}=5.5 \pm 3 k_{\mathrm{B}} T$.
\end{abstract}

\begin{abstract}
Forces between curved mica surfaces across a brine swollen lamellar phase (CTAB/hexanol/Brine) have been measured. The bilayers oriented spontaneously parallel to the mica surfaces. The force-distance curves show regimes where brine is continuously expelled from between the lamellae, and regimes in which bilayers are ejected over a few seconds. We have investigated the effect on the forces of moving in the phase diagram at constant bulk repeat distance, as well as along the dilution line. We found that the force necessary to squeeze out bilayers becomes smaller as the system approaches the transition to the bicontinuous phase $\left(\mathrm{L}_{\alpha} / \mathrm{L}_{3}\right)$, i.e. on increasing the hexanol content. Dynamic effects are observed and may be explained in terms of hydrodynamic coupling of the bilayers with the solvent. They may produce surprising behaviors: on decompressing the phase, lamellae are systematically ejected. The equilibrium state of the system is discussed. The data is consistent with the $1 / d^{2}$ profile calculated by Helfrich for the undulation forces [1]. Least square fits of the data to $1 / d^{2}$ gives bilayer curvature modulus values of the order of $5.5 \pm 3 k_{\mathrm{B}} T$.
\end{abstract}

(*) URA 1306 du CNRS, laboratoire associé aux Universités Paris 6 et Paris 7. 


\section{Introduction.}

The forces between solid surfaces across liquid media have received much attention in the past twenty years [2]. Dispersion forces, electrostatic double-layer interactions [3], and steric interactions in the presence of macromolecules [4] are well understood. The forces due to the structure of the liquid near the surfaces have been measured in a number of cases [5-7].

Forces have also been measured with the solid surfaces coated by a lipid bilayer [8], giving information on the interaction between model biological membranes. These forces have been found to be somewhat different from those measured between free lipid bilayers under osmotic pressure [9]. It is now accepted that the difference arises from the fact that bilayers have thermal fluctuations which take the form of undulations. When those undulations are restricted by two walls, the entropy decrease gives rise to a repulsion of a steric nature between these walls and the bilayer(s). Helfrich [1] was the first to predict this effect and to calculate the interaction energy due to these undulations. For lipid bilayers, that have a bending elastic coefficient $k_{\mathrm{c}}$ of the order of $50 k_{\mathrm{B}} T$, these forces are smaller than Van der Waals forces, but of the same order of magnitude [1]. Other bilayers can have a $k_{\mathrm{c}}$ of the order of $k_{\mathrm{B}} T$ and these forces then become determinant.

For instance, the stability of swollen lamellar phases, which can have repeat distances of up to several hundreds of $\mathrm{nm}$, results from this undulation force [10-11]. These phases are in general a ternary mixture of surfactant, alcohol (which lowers $k_{\mathrm{c}}$ ) and solvent (brine or oil). The undulation forces have been measured by small angle $X$-ray scattering and by light scattering [12-14]. In the present paper we present the first force measurements obtained by confining one or several lamellae of a brine swollen lamellar phase, CTAB/hexanol/brine, between two mica surfaces. Forces between mica surfaces across an oil swollen lamellar phase have been measured by P. Kekicheff and H. K. Christenson [15].

Previous force measurements on lamellar systems between mica surfaces, performed with hydrated egg lecithin, revealed lipid bilayers oriented parallel to the surfaces [16]. The surfaces could not be smoothly brought closer together but only by jumps, indicating that the bilayers are squeezed out from between the surfaces. To squeeze out one bilayer required the formation of a defect whose geometry could not be completely defined. One aim of this work is to study the formation of defects in the lamellar phase.

\section{Materials and methods.}

Hexadecyl-trimethylammonium bromide (CTAB) is $99 \%$ pure A.R., and sodium chloride is suprapure, both purchased from Merck and used without further purification. Hexanol is $99 \%$ pure from Alfa Products (U.S.A.). Water is degassed miliQ-Organex.

The temperature at which the forces are measured is $22 \pm 0.5^{\circ} \mathrm{C}$.

FORCE MEASUREMENT APPARATUS. - In this paper, $D$ is the distance between the mica surfaces, $d_{0}$ is the bulk repeat distance of the lamellar phase and $d$ is the distance between adjacent bilayers confined between the mica surfaces.

The force $F$ between two molecularly smooth curved mica surfaces as a function of distance $D$ is measured using the technique developed by $\mathbf{J}$. N. Israelachvili and extensively described in [3]. The main part of the system is a calibrated three stage translation device, composed of a micrometric unit coupled with a differential spring system (accuracy $1 \mathrm{~nm}$ ) and a piezoelectric tube. One mica sheet is fixed to the piezoelectric tube and the other one is fixed to a leaf spring attached to the differential spring system. The force is deduced from the measured flexion of this leaf spring. The distance $D$ between the mica surfaces, which depends both on the force and the position of the translation units, is measured by multiple 
beam interferometry to $0.1-0.2 \mathrm{~nm}$. The surfaces are set in a crossed cylindrical geometry which is equivalent to that of a sphere/plane when $D \ll R$ where $R$ is the radius of curvature of the surfaces. The interaction energy $E(D)$ between flat surfaces per unit area is deduced from the measured force $F(D)$ between curved surfaces by the Derjaguin approximation: $F(D)=2 \pi R E(D)$. In the present paper, the force/distance curves will be plotted as $F / R$, expressed in $\mu \mathrm{N} / \mathrm{m}$, versus $D$.

FORCES MEASUREMENT PROCEDURE. - The contact between the mica surfaces, obtained in air, is used as the $D=0$ reference. The surfaces are immersed in the swollen lamellar phase $\left(\mathrm{L}_{\alpha}\right)$ and left a few $\mu \mathrm{m}$ apart for 90 minutes. In order to immerse the surfaces, a small cell attached to the different spring system is used, which requires $10 \mathrm{ml}$ of solution. Then the surfaces are brought to smaller distances $(400-800 \mathrm{~nm})$, and left 30 minutes while the thermal distance drift is measured. This drift is in general constant and averages over all the experiments to $0.5 \mathrm{~nm} / \mathrm{min}$. In general, with this technique, the forces are measured with back and forth changes of distance $D$, using the calibrated piezoelectric tube ; however, we have observed that this method produces the ejection of bilayers much more often than using monotonic movements (i.e. without reversing the movement) consequently reducing the domains over which the force is at constant bilayer number. This is why, in a run, the forces are measured while the surfaces are, with the differential spring system, monotonically brought from the starting distance down to a small distance, and then separated with a monotonic movement to large distances, while continuing to measure the forces. At each distance, 90 seconds relaxation are allowed before measuring the force and moving to the next point.

For each sample composition, one to three experiments are performed. For each curve given, similar behavior was observed on three separate runs.

In order to avoid possible evaporation of the hexanol that would shift the phase to another point (this may be critical as shown in the results section), the experiments did not last more than about 6 hours.

The phase is filtered (Milipore GS $0.22 \mu \mathrm{m}$ ) prior to each experiment. The $\mathrm{L}_{\alpha}$ phase is birefringent and this is always checked with crossed polarizers before and after each experiment.

The results indicate that the lamellae are parallel to the mica surfaces. This orientation is confirmed by the following method : cells made of mica sheets separated by $125 \mu \mathrm{m}$ mylar spacers, and in which the mica birefringence is compensated, are filled with the $\mathrm{L}_{\alpha}$ phase. The observation with a polarizing microscope shows one homeotropic domain, with defects on the edges of the cell.

\section{Phase diagram.}

The phase diagram of CTAB, hexanol and brine $(0.34 \mathrm{M})$ in the brine rich domain at $21{ }^{\circ} \mathrm{C}$ is shown in figure 1 ; as it is very sensitive to the amount of alcohol, extreme care is taken in order to ensure the maximum accuracy in the determination of the transition lines. Nevertheless, the transition between the lamellar $L_{\alpha}$ and the micellar $L_{1}$ phase is only approximative (the dotted line in Fig. 1), because the equilibrium is not reached after one month ; for comparison, the equilibrium is obtained in a few minutes to a few hours at higher hexanol contents.

The lamellar structure is determined by observation, with a polarizing microscope, of typical focal conic defects. This lamellar phase can be diluted to repeat distances of a few hundred nm.

The phase diagram shown in figure 1 is very similar to those obtained by other authors with 


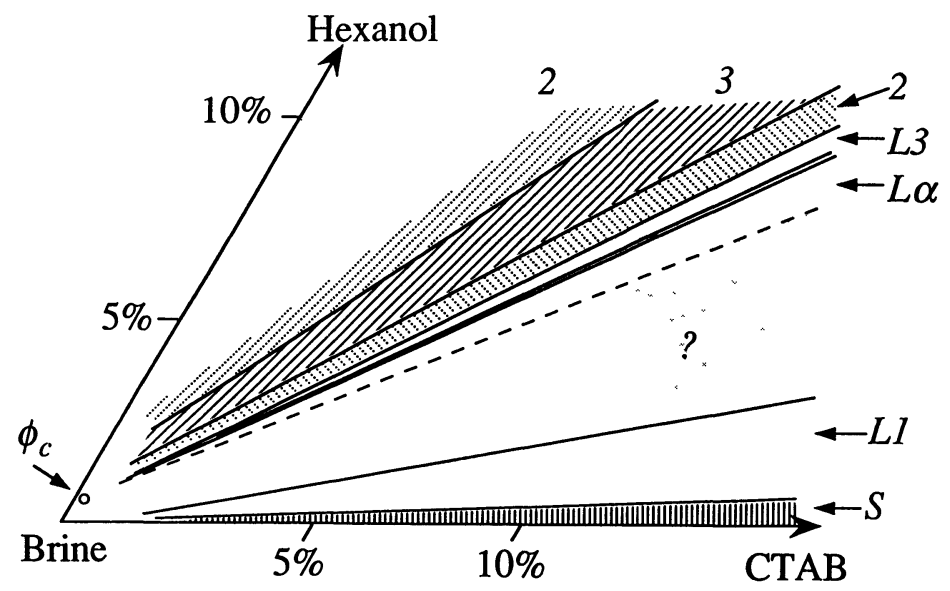

Fig. 1. - Phase diagram of $\mathrm{CTAB} /$ hexanol/brine $(\mathrm{NaCl} 0.34 \mathrm{M})$ at $21{ }^{\circ} \mathrm{C}$ (linear scale) : « $\mathrm{S} »$ : solid CTAB ; " L1 » : micellar phase ; " ? : not in equilibrium after one month ; " L $\alpha$ » : lamellar phase ; «L3»: disordered phase (see text); « 2 » : diphasic domain; « $3 »$ : triphasic domain ; « $\phi_{\mathrm{c}} »$ : composition of the continuous phase (see text).

cetylpirydinium chloride (CPCl), hexanol and brine [17]; furthermore, CTAB is similar to $\mathrm{CPCl}$, so we assume that the phase in equilibrium with $\mathrm{L}_{\alpha}$ at higher amounts of hexanol has the same structure as the $\mathrm{L}_{3}$ phase depicted by G. Porte et al. : a bicontinuous and disordered phase where the bilayers are locally arranged in a cubic structure [18].

DETERMINATION OF THE BULK REPEAT DISTANCE $d_{0}$ OF THE PHASE. $-d_{0}$ is determined from an estimated thickness of the lamellae, $2.2 \mathrm{~nm}$ (see next section), the known composition of the phase, and the composition of the continuous phase. The latter is obtained from an extrapolation to infinite dilution (see the phase diagram).

\section{Results.}

All the force-distance measurements (Figs. 2 and 3) show continuous regimes and regimes where the system jumps, on a time scale of seconds, towards a new stable distance. In a continuous regime, water is expelled from between the bilayers; when there is a jump, bilayers plus some water are ejected from between the mica surfaces.

It is evident that the $\mathrm{L}_{\alpha}$ phase compressed between the mica surfaces is metastable during the experiment : the measured force is not a single-valued function of distance (Fig. 3f); furthermore, for some points in the phase diagram, bilayers are systematically squeezed out upon decompressing the phase (i.e. acting to separate the surfaces) (Fig. 3f) : when the differential spring system is moved in order to separate the surfaces, the distance $D$ between the mica surfaces should increase; however, if bilayers are ejected during this movement, the compression of the elastic medium decreases and the leaf spring, by which one mica sheet is fixed to the differential spring system, unbends ; the distance $D$ between the mica surfaces is thus decreased!

The real thermodynamic equilibrium is not reached : the number of squeezed bilayers is not the number at equilibrium, with the most striking demonstration in figure 3f. This metastability has the drawback that the jumps, from one metastable state to another, occur according to some probability law: this explains the apparent irregularity of the forcedistance curves although the general pattern is reproducible. However this metastability 

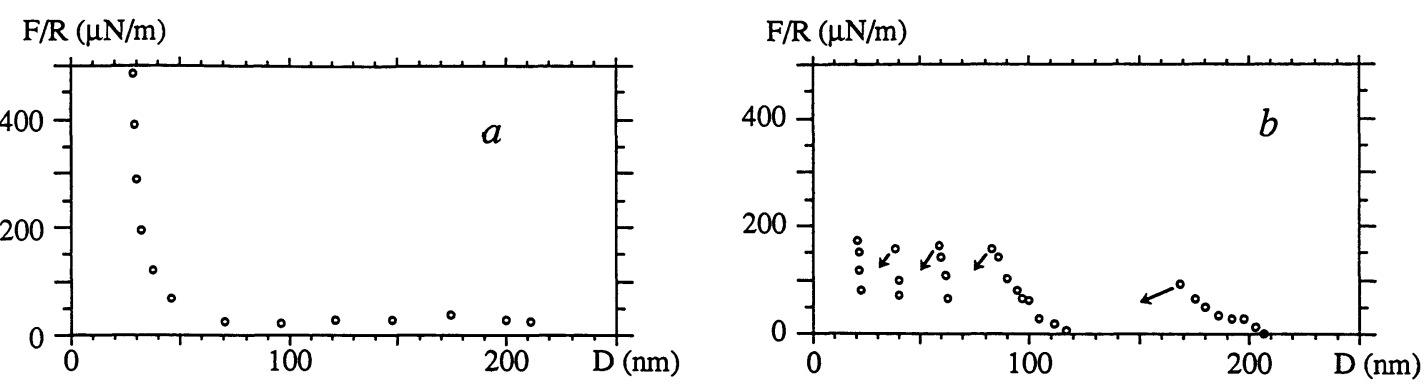

Fig. 2. - a, b : force/distance measurements performed while bringing the surfaces closer, with the phases a and $\mathrm{b}$ mentioned in the table I for which $d_{0}=23 \mathrm{~nm}$.

$\mathrm{F} / \mathrm{R}(\mu \mathrm{N} / \mathrm{m})$

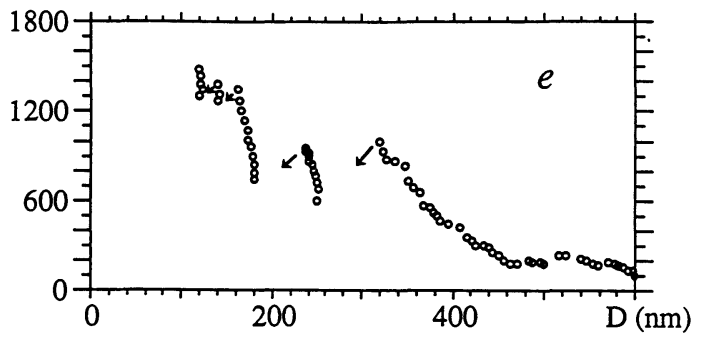

$\mathrm{F} / \mathrm{R}(\mu \mathrm{N} / \mathrm{m})$

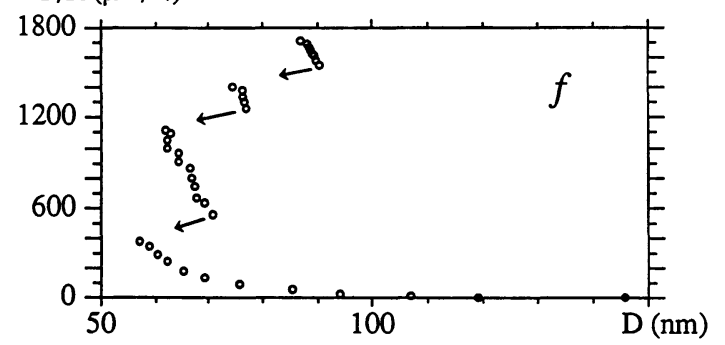

Fig. 3. - e : Force/distance measurements performed while bringing the surfaces closer with the phase e mentioned in the table I, for which $d_{0}=23 \mathrm{~nm}$. $\mathrm{f}$ : force measured on decompressing the lamellar phase (phase e) : when the differential spring system is moved in order to separate the surfaces, the distance $D$ between the mica surfaces should increase ; as bilayers are ejected during this movement, the compression of the lamellae decreases and the leaf spring, by which one mica sheet is fixed to the differential spring system, unbends; the distance $D$ between the mica surfaces is thus decreased.

allowed us to obtain force-distance curves over larger distance domains, at a fixed number of bilayers. An idealized thermodynamic equilibrium would imply for instance that the number of bilayers between the surfaces would be the closest integer from $D / d_{0}$. The distance domains at constant bilayer number would then be restricted to $d_{0}$.

Although the system is not at thermodynamic equilibrium, the bilayers are at mechanical and hydrodynamic equilibrium : all stresses due to hydrodynamic movements (inertia and viscosity) after a displacement of the mica plates have relaxed. We have observed that after a large perturbation, such as a jump, the stresses relaxed to equilibrium in typically 2 minutes. In the regime where the force is continuous the distance between two points is about $7 \mathrm{~nm}$. For such a small perturbation, 90 seconds should be generous for the relaxation of the tension possibly created on the bilayers during the movement, and thus for the restoration of the undulations to their thermodynamic equilibrium level. This delay is also sufficient for the relaxation of viscosity effects [23]. Because of the presence of drifts in the system, the time allowed for equilibration must be limited and is set at this 90 seconds level between two points.

We have also noticed that no jump towards larger distances, that would correspond to a discrete number of bilayers coming between the mica surfaces, is ever observed on decompressing the lamellar phase. 
EFFECT OF MOVING IN THE PHASE DIAGRAM AT CONSTANT BULK LAMELLAR SPACING $d_{0}$. - This effect has been studied for a bulk repeat distance $d_{0}=23 \mathrm{~nm}$ (Figs. 2 and 3 ). Several phases have been made with this repeat distance, but with a different alcohol content (see Tab. I). They can be represented on the phase diagram as points which are on a line perpendicular to the axis of the lamellar phase region. Each of these phases is studied by forces measurements.

Table I. - Composition of the different studied lamellar phases at $23 \mathrm{~nm}$ repeat distance. $F_{\mathrm{J}}$ is the average force at which bilayers are squeezed out on compression.

\begin{tabular}{|c|c|c|c|c|}
\hline & $\begin{array}{c}\text { Hexanol } \\
\% \mathrm{w} / \mathrm{w}\end{array}$ & $\begin{array}{c}\text { CTAB } \\
\% \mathrm{w} / \mathrm{w}\end{array}$ & $\begin{array}{c}\text { Brine } \\
\% \mathrm{w} / \mathrm{w}\end{array}$ & $\begin{array}{c}F_{\mathrm{J}} \\
(\mu \mathrm{N} / \mathrm{m})\end{array}$ \\
\hline $\mathrm{a}$ & 4.55 & 5.56 & 89.89 & 0 \\
$\mathrm{~b}$ & 4.54 & 5.57 & 89.89 & $110 \pm 30$ \\
$\mathrm{c}$ & 4.50 & 5.61 & 89.89 & $120 \pm 50$ \\
$\mathrm{~d}$ & 4.45 & 5.66 & 89.89 & $400 \pm 200$ \\
$\mathrm{e}$ & 4.39 & 5.72 & 89.89 & $1100 \pm 400$ \\
\hline
\end{tabular}

At the border with the diphasic region (lamellar $\mathrm{L}_{\alpha}$ /bicontinuous $\mathrm{L}_{3}$ ) the force-distance curves are continuous within the force resolution of the apparatus, and no jump is observed (see Fig. 2a). The surfaces can be brought together to a small separation until one or a few bilayers are trapped; at this point, the force increases to large values $(2-10 \mathrm{mN} / \mathrm{m})$ without any jump occurring (this may be due to the strong ordering effect of the mica surfaces). Even when the lamellae are ejected at zero force, there can be some trapped layers between the surfaces, as shown in figure $2 \mathrm{a}$.

Decreasing the alcohol content from $4.55 \%$ to $4.54 \%$ i.e., going slightly more inside the lamellar region (see Fig. 2b), the force-distance curves are no longer continuous, and the mica surfaces come together by jumps alternating with continuous movements. Over a distance domain of at least $150 \mathrm{~nm}$, the following regime is observed : as the repulsive force reaches some order of magnitude $F_{\mathrm{J}}$, the system jumps to a new state at another distance ; one or several bilayers are squeezed out. On bringing the surfaces closer, this process reoccurs until the force becomes large with no further bilayer ejected.

Figures 2, 3 and table $I$ show that $F_{\mathrm{J}}$ increases on decreasing the alcohol content of the mixture.

EFFECT OF Diluting THE LAMELlaR PHASE. - At smaller repeat distances (17, 20.5 and $22 \mathrm{~nm}$ ), the same behavior as that shown in figures 2 and 3 is observed.

On moving along the dilution line towards a bulk repeat distance $d_{0}$ equal to $32 \mathrm{~nm}$, long distance forces are no longer observed. It proved very difficult to find a point in the phase diagram, at this repeat distance, for which there is a non-zero force above $D=70 \mathrm{~nm}$ micamica distance. The results obtained with a phase having the composition $4.20 \% \mathrm{CTAB}$, $3.32 \%$ hexanol, $92.48 \%$ brine, $\left(d_{0}=32 \mathrm{~nm}\right)$ are shown in figure 4 : several force-distance curves are plotted corresponding to different runs with a different number of lamellae trapped between the mica surfaces. The curves of figure $4 \mathrm{~b}$, starting from the right correspond to numbers of free bilayers differing by 1 , from 0 to 5 . The distance $d$ between the bilayers under the compression of $500 \mu \mathrm{N} / \mathrm{m}$ can be estimated from the distances between the curves of figure 4. This value averages to $2.8 \mathrm{~nm}$. 

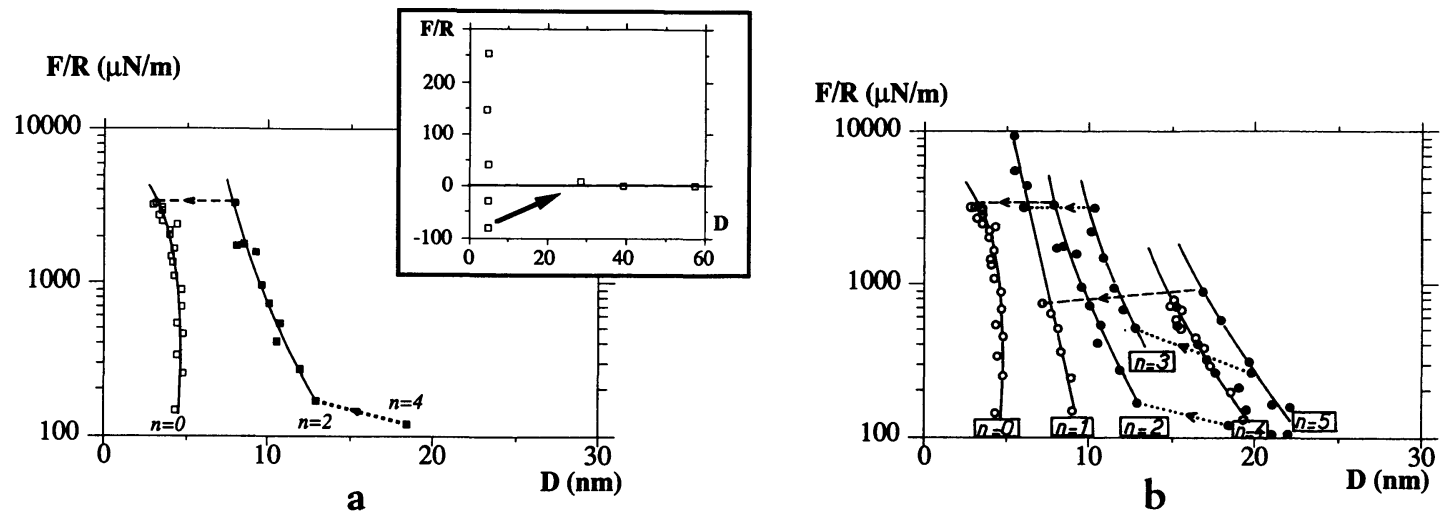

Fig. 4. - Force/distance measurements with the diluted phase : $d_{0}=32 \mathrm{~nm}$. Filled points correspond to forces measured on compression, and open points to forces measured on decompression. Dashed and dotted lines with arrows indicate the jumps, corresponding to the ejection of lamellae, that occurred respectively on decompression and on compression. Solid lines are guide for the eyes. These lines are numbered according to the number of free bilayers. (a) One run in which the forces are measured on compression starting with 4 free lamellae and continuing with 2 . On decompression, two further lamellae are ejected and some adhesion is measured (shown in the insert). (b) Several runs (including (a)) of force/distance measurements.

In the quantitative analysis of the forces done in the next paragraphs, we need a value of the bilayer thickness. In the CPCl-hexanol-brine lamellar system, the measured thickness of a bilayer is about $2.2 \mathrm{~nm}$ [19]. CTAB is slightly smaller than $\mathrm{CPCl}$ and conversely, we should take into account the hydration thickness. We will then take a value of $2.2 \mathrm{~nm}$ for the bilayer thickness. This is coherent with the $d=2.8 \mathrm{~nm}$ value obtained at $F / R=500 \mu \mathrm{N} / \mathrm{m}$; at this compression, most of the water is ejected except a few Ångströms. In any case, the undulation forces analysis is relatively insensitive to the exact value (see $\S$ Undulation forces).

We note that the only jumps observed with this dilute phase consist of jumps of 2 or 4 lamellae.

All the curves in figure $4 \mathrm{~b}$ indicate a repulsive interaction, except the first curve at the left (also shown in Fig. 4a) which corresponds to no undulating bilayer trapped between the surfaces. This curve shows a small attraction (see the insert). The next curve, with one free bilayer, does not have any attractive regime: the extra-repulsion resulting from the introduction of one or several free bilayers is an experimental demonstration of the existence of repulsive undulation forces.

DYNAMIC EFFECTS. - Some important dynamic effects have been systematically observed during the runs. Each time we reverse the movement of the differential spring system, after having compressed several bilayers in a metastable state, one jump closer is immediately observed, showing that bilayers are ejected on decompression (see for example Fig. 4). In the phase $d$ and e, more jumps closer are observed (Fig. 3f) on continuing to move the differential spring system in order to separate the surfaces (i.e. during the decompression, the system jumped from one metastable state to another in a stepwise manner ; each state corresponds to a different number of trapped bilayers). The stability of the bilayers is decreased on decompression. The possibility of a mechanical artifact has been considered : a shear could be produced in reversing the movement of the differential spring system but the same jumps are observed using the piezoelectric tube for the reversal. 
Another dynamic effect is observed: after each jump, the force relaxed towards larger values. A statistical average of the difference between the relaxed and non-relaxed force is $+60 \mu \mathrm{N} / \mathrm{m}$; the duration of this relaxation is 2 minutes. This effect is not obvious on the scale of the curves given in figures 2,3 and 4 , but it is clear in the actual measurements.

Adsorbed bilayers on the mica surfaces. - At distances of a few $\mathrm{nm}$, the forces always became large, showing the presence of an adsorbed bilayer on each surface, whose thickness $e$ could only be estimated from the mica-mica distance : $e=2.3 \pm 0.7 \mathrm{~nm}$. Only an estimation of the thickness may be obtained because : i) we do not have any reliable zero distance reference (only contact in air is used as $D=0$; in this contact, there are always $0.5-1.0 \mathrm{~nm}$ impurities that go into solution on immersing the surfaces [3]), ii) some water could remain between the bilayers and iii) the bilayers may be compressible.

\section{Analysis of the data.}

VALIDITY OF THE DERJAGUIN APPROXIMATION. - The validity of the Derjaguin approximation in the case of nematic liquid crystals has been discussed in reference [5]. The same discussion would apply to smectics if there are no edge dislocation. However one may argue that it is not valid for our system, on the grounds that in the crossed cylinder geometry there is, near the mica surfaces, a distribution of edge dislocation loops in the lamellar phase ; these dislocations, which have been observed by F. Nallet and J. Prost in reference [22], have a line tension which, in this geometry would give a contribution to the repulsive force between the mica surfaces. However, we have observed that on separating the surfaces, the forces always decays to a baseline on a $100 \mathrm{~nm}$ scale, in general much more quickly. This shows that the putative contribution of the dislocations to the force is negligible or constant with distance.

FORMATION OF A DEFECT IN THE LAMELLAR PHASE: GEOMETRY AND ENERGY CONSIDERATIONS. - For a jump to occur in our crossed-cylinder geometry, an edge dislocation loop in the liquid crystal phase must be created. The most salient feature of the data is that the jumps occur at a smaller $F / R$ (i.e. require less and less energy) as we get close to the $\mathrm{L}_{\alpha} / \mathrm{L}_{3}$ transition line (see Tab. I). This is expected from defects that have the same geometry as the basic structure element of the bicontinuous phase.

Several topologies for the defects created during a jump are possible, corresponding to different values of the Burgers vector. The 3 possible topologies are represented in figure 5, one with a Burgers vector of 2 , which has the same topology as is generally assumed for the fusion of biological membranes (Fig. 5a), and two with a Burgers vector of 1, one corresponding to the fusion of two layers into one, sometimes called hemi-fusion (Fig. 5b), and one corresponding to a hole in the bilayer (Fig. 5c). Holes in bilayers will be created only if the compression from the mica surfaces generates a high tension on the bilayers. As we have observed ejection of lamellae also on decompression, holes have to be ruled out for explaining the jumps during the runs. We are now left with the two other possibilities. A thermodynamical argument favors the occurrence of Burgers vector 2 edge dislocation loops : their geometry is identical to the basic structure element of the $\mathrm{L}_{3}$ bicontinuous phase, and the energy of creation of such defects should decrease on getting closer to the $\mathrm{L}_{\alpha} / \mathrm{L}_{3}$ transition, as is observed (see Tab. I). Furthermore, where we can determine with sufficient accuracy the number of layers ejected in a jump (Fig. 4), only multiples of two are observed. Although these arguments are not a formal proof that all the jumps occur by the formation of edge dislocation loops with Burgers vector a multiple of 2 , we can say with good confidence that we have this geometry. 

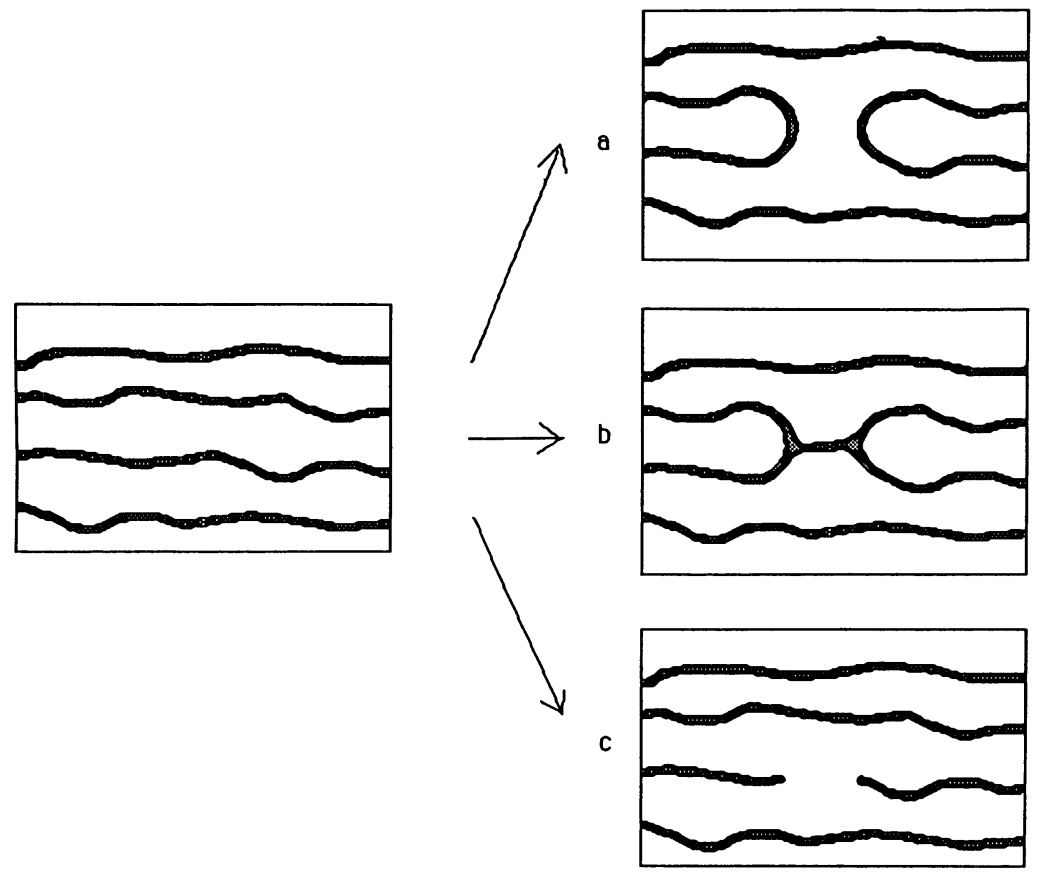

Fig. 5. - The three different possible geometries of the defects generated during a jump : a) the total fusion has a Burgers vector of 2, and b) and c) a Burgers vector of 1. In b) two lamellae fuse into one. c) corresponds to a hole in the bilayer.

DYNAMIC EFFECTS. - The large intrinsic roughness of the undulating bilayers can provide a consistent explanation of the dynamic effects. The rougher the bilayers are, the stronger their hydrodynamic coupling with water. The bilayer roughness is very sensitive to the tension, and can vary dramatically with this tension.

Relaxation of the force after a jump. - When there is a jump, one or several bilayers plus some water leave the space between the mica surfaces and the slipping, through the hydrodynamic coupling exerts a tension on the adjacent layers : the undulations are thus reduced, and so are the undulation forces. When this tension relaxes to zero, the thermal undulations are restored, and the forces relax up to their equilibrium value. We observe a characteristic time for the relaxation of bilayers tension of the order of 2 minutes. Nevertheless a change of bilayer thickness under compression may not be excluded as a contribution to this effect.

Bilayer ejection occurring on decompression. - The solvent/bilayer hydrodynamical coupling may also provide a possible explanation of this effect.

When the surfaces are brought closer together in the continuous regime, a small tension on the bilayers is also created through the hydrodynamical coupling and relaxes down after the flow has stopped.

The situation is very different when we act to separate the surfaces (decompression) : water is sucked in between the bilayers inducing through the hydrodynamic coupling a lateral pressure on the bilayers : this will strongly enhance the undulations and can produce buckling instabilities [24]. Similar effects have been observed on thermotropic smectic liquid crystals $[27,28]$. The strong increase of the undulations, and/or buckling may lead to an ejection of 
bilayers, if they are compressed in a metastable state, resulting in a jump towards a smaller mica/mica distance $D$.

UNDULATION FORCES. - In order to analyse the measured force in terms of undulation forces, the other surface interactions must be subtracted or negligible. When the distance $d$ between bilayers is below $5 \mathrm{~nm}$, the hydration force and the screened electrostatic repulsion (the Debye length for $\mathrm{NaCl} 0.34 \mathrm{M}$ is about $0.5 \mathrm{~nm}$ ) may not be negligible. Therefore, we analyse the force data only when $d$ is above $5 \mathrm{~nm}$, i.e. only when the undulation forces are determinant. These forces were predicted by Helfrich [1]: when the undulations are restricted, the entropy decrease produces a repulsive interaction. He calculated the corresponding free energy $E$ of steric interaction per unit area of membrane in a multilayer system :

$$
E(d)=\frac{3 \pi^{2}}{128} \frac{\left(k_{\mathrm{B}} T\right)^{2}}{k_{\mathrm{c}} d^{2}}
$$

where $k_{\mathrm{c}}$ is the bending elastic modulus (or rigidity) of one bilayer, $k_{\mathrm{B}}$ the Boltzmann constant, $T$ the thermodynamic temperature and $d$ the distance between two adjacent membranes. He also did the calculation in the case of one bilayer confined between two walls, where the result differs from the above expression only by a numerical factor : $3 \pi^{2} / 128$ is replaced by $3 / 32$ [1].

As the lamellar phase compressed between the mica surfaces is in a metastable state, one may ask whether Helfrich's equation applies: there is only more bilayers between the surfaces than at thermodynamic equilibrium; this metastability does not change the characteristics of the bilayers which are still undulating according to the Boltzmann law. In other words, and as discussed in the previous section, the characteristic times of relaxation are much lower than the characteristic time of our measurements which in turn is much lower than the lifetime of the metastable equilibrium when $F<F_{\mathrm{J}}$. Therefore, our data can be analysed in terms of Helfrich's theory.

Unfortunately, our data does not span a sufficiently large scale of force and distance $d$ to accurately check the exponent of $d$ in the interaction energy : at large $d$ the Helfrich force is too weak to be measured with our apparatus and at small $d$, other interactions (Van der Waals, electrostatic double-layer, hydration forces) may no longer be negligible, and jumps may occur, changing the number of lamellae. We will restrict our analysis to fitting our data with Helfrich's results in order to deduce the bending elastic modulus (or rigidity) $k_{\mathrm{c}}$ of one bilayer. Although we have a finite number of lamellae between the mica surfaces which may introduce some error on the determination of $k_{\mathrm{c}}$, we will use the above equation, in the analysis that follows, with one correction : the distance, at a given force, between an undulating bilayer and a non-undulating bilayer adsorbed on the mica is smaller than that between two undulating bilayers $d$, and will be taken here to $0.75 d$; the exact value is unnecessary as the uncertainty on the bilayer number is large.

In our experiments we measure $F(D) / R=2 \pi E_{\mathrm{t}}(D)$ according to the Derjaguin approximation, where $E_{\mathrm{t}}$ is the free energy of interaction per unit area between plane parallel mica surfaces at distance $D$. For $n$ fluctuating bilayers between the mica surfaces, $E_{\mathrm{t}}(D)=n E(d)$, when the effect of edge dislocation loops is neglected. As the relation between $D$ and $d$ is $D \approx(n+2) e+(n+0.5) d$, where $e$ is the thickness of the bilayer, the measured force for a constant number of lamellae is given by

$$
\frac{F}{R}(D)=\frac{6 \pi^{3}}{128} \frac{\left(k_{\mathrm{B}} T\right)^{2}}{k_{\mathrm{c}}} \frac{n(n+0.5)^{2}}{[D-(n+2) e]^{2}} .
$$


The number of lamellae trapped between the mica plates must be known. We cannot, in general, directly count the lamellae from our data but we are able to obtain a reliable value for the number of lamellae in a force-distance curve from the coherence of several parameters which are estimated, not accurately determined :

- number of lamellae at the smallest distance,

- number of lamellae at the starting (largest) distance,

- number of lamellae before and after each jump, deduced from the jump length. e.g. for a jump starting from $D_{1}$ with $n$ lamellae to $D_{2}$ with $n-2$ lamellae, $n$ is deduced from : $D_{1} /(n+1) \approx D_{2} /(n-1)$.

The typical uncertainty on the number of bilayers is about $10 \%$.

Equation (2) fits the data fairly well : some examples of force curves are plotted in figure 6 as a function of $d^{-2}$; only data between two consecutive jumps are plotted ; the straight line is the least square fit. From the slope of this line the bending elastic modulus $k_{\mathrm{c}}$ of one bilayer is derived but the uncertainty is rather large : from equation (2) it can be seen that the force is roughly proportional to the third power of the number of bilayers, so the obtained values of $k_{\mathrm{c}}$ are very sensitive to the estimated number of lamellae: this is the main source of uncertainty for $k_{\mathrm{c}}$.
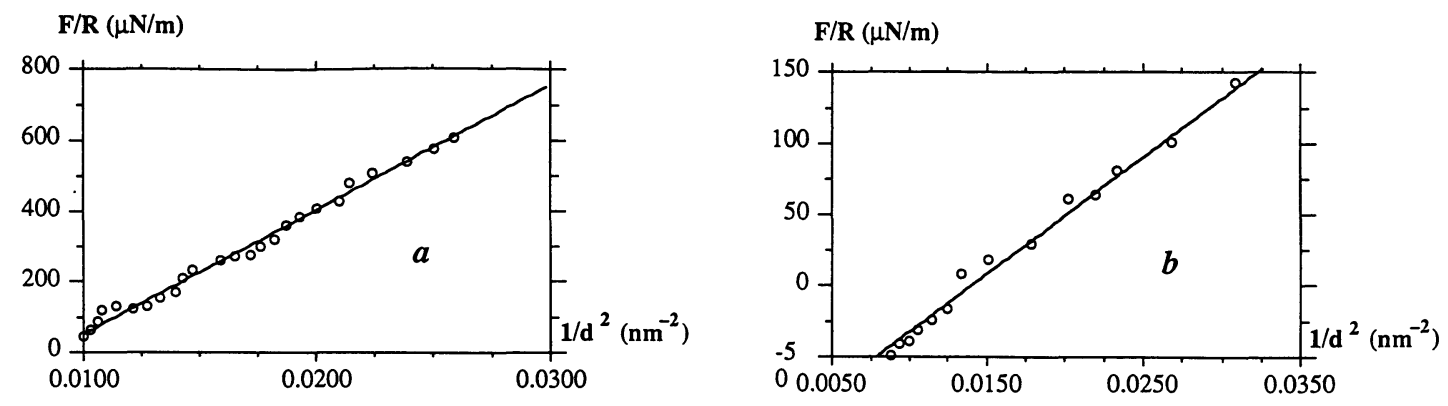

Fig. 6. - Least square fits to Helfirch's formula of portions of the forces; $6 a$ shown in figure $2 b$ ( 32 free bilayers, $\left.k_{\mathrm{c}}=5.3 k_{\mathrm{B}} T\right)$, and $6 \mathrm{~b}$ not shown $\left(10\right.$ bilayers, $\left.k_{\mathrm{c}}=7.5 k_{\mathrm{B}} T\right)$.

For phases with $d_{0}=23 \mathrm{~nm}$ bulk repeat distance and different hexanol contents, values of $k_{\mathrm{c}}$ are listed in table II. At $4.55 \%$ hexanol concentration, the $\mathrm{L}_{\alpha}$ phase is very close to the transition with $\mathrm{L}_{3}$ phase : the transition occurs when the temperature is decreased by only a few degrees. No significant variation of $k_{\mathrm{c}}$ can be seen : this implies that the transition

Table II. - The composition of the phases $a, b, c, d, e$ are given in table $I ; k_{\mathrm{c}}$ is the average value of the bending elastic modulus obtained from all the force-distance curves.

\begin{tabular}{|c|l|}
\hline Phaseat23 nm repeat distance & $k_{\mathrm{c}} / k_{\mathrm{B}} T$ \\
\hline $\mathrm{a}$ & $5.5 \pm 2.5$ \\
$\mathrm{~b}$ & $6.0 \pm 3$ \\
$\mathrm{c}$ & $5.0 \pm 3.5$ \\
$\mathrm{~d}$ & $7.0 \pm 3$ \\
$\mathrm{e}$ & $7.0 \pm 3.5$ \\
\hline
\end{tabular}


$\mathrm{L}_{\alpha} / \mathrm{L}_{3}$ is not driven by the variation of $k_{\mathrm{c}}$ but probably by the variation of the bending elastic modulus associated with the Gaussian curvature, as predicted by G. Porte [20].

There is no evidence of any variation of $k_{\mathrm{c}}$ with the number of lamellae or with the dilution ratio at low dilution i.e. for a mean repeat distance at equilibrium below $25 \mathrm{~nm}$. A statistical average of $k_{\mathrm{c}}$ on all force data obtained at low dilution gives : $k_{\mathrm{c}}=5.5 \pm 3 k_{\mathrm{B}} T$. This is to be compared with values obtained in other phases by light or X-ray scattering technique : $k_{\mathrm{c}}=0.5$ to $2 k_{\mathrm{B}} T$ for sodium dodecylsulphate/pentanol/water bilayers swollen in dodecane $[25,26]$. It is also interesting to compare this value with the rigidity $k_{\mathrm{c}}^{\prime}=0.4 k_{\mathrm{B}} T$ measured by ellipsometry for a monolayer of CTAB and butanol in a microemulsion [21] : the rigidity of a fluid bilayer is twice that of a monolayer, and it increases very rapidly with the chain length of the surfactant or cosurfactant (hexanol is longer than butanol).

Forces at higher dilution (bulk repeat distance $d_{0}$ of $32 \mathrm{~nm}$ ). - The distance $d$ between bilayers at which forces started to be measurable are much smaller (below $3 \mathrm{~nm}$ ) than those for lower dilutions (i.e. $d_{0} \leqslant 23 \mathrm{~nm}$ ); this is checked at various hexanol contents. In this case Van der Waals, electrostatic double-layer and hydration forces may not be negligible, and we cannot fit the force curves with Helfrich's formula. However the total repulsive forces are weaker than for phases at low dilutions, and we do not understand why : the composition of a bilayer should not change along a dilution line, therefore $k_{\mathrm{c}}$ and the undulation forces should not vary so much. In order to understand the behavior at this dilution, we plan to measure the forces at intermediate dilutions.

COMPARISON WITH THE RESULTS OF KÉKICHEFF AND CHRISTENSON [15]. - There are major differences between their results and ours :

- they observed a force oscillating with distance superimposed on an attractive background and their system seems to be in thermodynamic equilibrium; we have not observed any attractive background, and our compressed phase is in a metastable state. This has allowed us to compress the bilayers at a higher level without ejecting them ;

- in their system, lamellae are squeezed out one by one while we observe defects of Burgers vector 2 ;

- on separating the surfaces after a long wait, they observed jumps towards larger distances; we did not wait before decompressing the lamellae between the surfaces and we obtained, after the ejection of a few metastable bilayers, a smooth force-distance curve decaying to zero.

These significant differences are not well understood. However there are major differences in the lamellar phase studied : their lamellar phase is a quaternary mixture swollen with oil (water $10.04 \%$, SOBS $9.23 \%$, 1-pentanol $12.78 \%$, n-decane $67.94 \%$ ) while ours is a ternary mixture swollen with brine.

\section{Conclusion.}

The swollen lamellar phase (CTAB/hexanol/brine) compressed between the mica surfaces is in a metastable state as regards the bilayer number. This has the advantage that much higher compressions can be reached at a constant lamellae number; furthermore, this allows us to measure the force at which lamellae are ejected. An unexpected consequence of this metastability is that bilayers can be squeezed out on decompression.

We have obtained another experimental demonstration of the existence of the undulation forces: without any free bilayer between the mica surfaces, we have an attractive force regime which disappears when we introduce a free bilayer.

Analyzing the data with Helfrich's theory, we have been able to obtain an estimation of the 
curvature elastic modulus of bilayers : $k_{\mathrm{c}}=5.5 \pm 3 k_{\mathrm{B}} T$. This rigidity does not significantly vary on moving the phase towards the $\mathrm{L}_{\alpha} / \mathrm{L}_{3}$ transition.

Our measurements have shown unambigously that the force necessary to eject bilayers decreases dramatically with increasing the hexanol content. The edge dislocation loops necessary for bilayers to be squeezed out have a Burgers vector of 2. Their topology is the same as the basic structure element of the $L_{3}$ phase.

The formation of a defect appears as the formation of a small element of the $\mathrm{L}_{3}$ phase in the lamellar phase. As the force necessary to form such a defect depends highly on the hexanol content and as $k_{\mathrm{c}}$ does not, our study supports the prediction of G. Porte [20] that the transition $\mathrm{L}_{\alpha} / \mathrm{L}_{3}$ is not driven by the variation of $k_{\mathrm{c}}$ but probably by the variation of the bending elastic modulus associated to the Gaussian curvature.

At high dilutions, the undulation forces decrease : this point remains unexplained and requires further investigation at intermediate dilutions.

Quantitative theory on the coupling between tension and undulations of the bilayers and hydrodynamics should be developped to provide a more accurate description of the observed dynamic effects. It would also be convenient to work with simpler systems such as binary mixtures or phases with non-ionic surfactants.

\section{Acknowledgments.}

We would like to acknowledge G. Porte, D. Langevin and J. Meunier for helpful discussions. Support is provided by the DRET, contract $n^{\circ} 88 / 1419$.

\section{References}

[1] Helfrich W., Z. Naturforsch. 33a (1978) 305.

Helfrich W., Servuss R.-M., Nuovo Cimento D 3 (1984) 137.

[2] Intermolecular and surface forces, IsRaelachVILI, J. N. (Academic Press, London) 1985.

[3] Israelachvili J. N., Adams G. E., Chem. Soc. Faraday Trans. I 74 (1978) 975.

[4] Klein J., J. Colloid Interface Sci. 111 (1986) 305.

[5] Horn R. G., Israelachvili J. N., Perez E., J. Phys. France 42 (1981) 39.

[6] IsRaelachVili J. N., Pashley R., Nature 306 (1983) 249.

[7] Christenson H., Horn R. G., Israelachvili J. N., J. Colloid Interface Sci. 88 (1982) 79.

[8] MARRA J., IsRaelachVili J. N., Biochemistry 24 (1985) 4608.

[9] Le Neveu D. M., Rand R. P., Parsegian V. A., Gingell D., Biophys. J. 18 (1977) 209.

[10] Leibler S., J. Phys. France 47 (1986) 507.

[11] For a brief review : Peliti L., Physica 140A (1986) 269.

[12] Safinya C. R., Roux D., Smith G. S., Sinha S. K., Dimon P., Clark N. A., Belloce A. M., Phys. Rev. Lett. 57 (1986) 2718.

[13] Safinya C. R., Sirota E. B., Roux D., Smith G. S., Phys. Rev. Lett. 62 (1989) 1134.

[14] Nallet F., Roux D., Prost J., Phys. Rev. Lett. 62 (1989) 276.

[15] Kekicheff P., Christenson H. K., Phys. Rev. Lett. 63 (1989) 2823.

[16] Terminassian-Saraga L., Perez E., Colloids and Surfaces 12 (1984) 213.

[17] Porte G., Gomati R., El haitami O., Appel J., Marignan J., J. Phys. Chem. 90 (1986) 5746.

[18] Porte G., Marignan J., Bassereau P., May R., J. Phys. France 49 (1988) 511.

[19] PORTE G., private communication.

[20] Porte G., Appel J., Bassereau P., Marignan J., J. Phys. France 50 (1989) 1335.

[21] Binks B. P., Meunier J., Abillon O., Langevin D., Langmuir 5 (1989) 415.

[22] Nallet F., Prost J., Europhys. Lett. 4 (1987) 307.

[23] Horn R. G., Hirz S. J., Hadzioannou G., Frank C. W., Catala J. M., J. Chem. Phys. 90 (1989) 6767. 
[24] Milner S. T., Joanny J.-F., Pincus P., Europhys. Lett. 9 (1989) 495.

[25] Roux D., Safinya C. R., Physics of Amphiphilic layers, Eds. J. Meunier, D. Langevin and N. Boccara (Springer-Verlag - Proceedings in Physics 21, Berlin) 1987.

[26] Nallet F., Roux D., Prost J., J. Phys. France 50 (1989) 3147.

[27] Delhaye M., Ribotta R., Durand G., Phys. Lett. 44A (1973) 139.

[28] Clark N. A., Meyer R. B., Appl. Phys. Lett. 22 (1973) 493. 